\title{
Photodissociation of neutron deficient nuclei
}

\author{
K. Sonnabend ${ }^{a}$, M. Babilon, J. Hasper, S. Müller, M. Zarza, and A. Zilges \\ Institut für Kernphysik, TU Darmstadt, Schlossgartenstr. 9, D-64289 Darmstadt, Germany
}

Received: 20 June 2005 /

Published online: 7 March 2006 - (C) Società Italiana di Fisica / Springer-Verlag 2006

\begin{abstract}
The knowledge of the cross sections for photodissociation reactions like e.g. $(\gamma, n)$ of neutron deficient nuclei is of crucial interest for network calculations predicting the abundances of the so-called $p$ nuclei. However, only single cross sections have been measured up to now, i.e., one has to rely nearly fully on theoretical predictions. While the cross sections of stable isotopes are accessible by experiments using real photons, the bulk of the involved reactions starts from unstable nuclei. Coulomb dissociation (CD) experiments in inverse kinematics might be a key to expand the experimental database for $p$-process network calculations. The approach to test the accuracy of the CD method is explained.
\end{abstract}

PACS. 25.20.-x Photonuclear reactions - 26.30.+k Nucleosynthesis in novae, supernovae and other explosive environments

\section{Introduction}

Most of the elements heavier than iron are produced by a sequence of neutron capture processes and $\beta$ decays. The $s$-process takes place during stellar burning phases and is characterized by low neutron densities $\left(n_{n} \approx\right.$ $\left.2-4 \cdot 10^{8} \mathrm{~cm}^{-3}\right)$ and temperatures $\left(T \approx 1-3 \cdot 10^{8} \mathrm{~K}\right)[1]$. Thus, the so-called $s$-process path is close to the valley of stability. In contrast, the $r$-process deals with high neutron densities $\left(n_{n}>10^{20} \mathrm{~cm}^{-3}\right)$ and temperatures $\left(T \approx 2-3 \cdot 10^{9} \mathrm{~K}\right)$ and is thought to occur in explosive scenarios like, e.g., supernovae [2,3].

However, several proton-rich isotopes between Se and $\mathrm{Hg}$ remain that cannot be produced during either of the processes. A complete list of these so-called $p$ nuclei can be found in $[4,5]$. These nuclides are also produced during explosive events lasting a few seconds at temperatures of about $2-3 \cdot 10^{9} \mathrm{~K}$. The lighter $p$ nuclei are thought to be produced by proton capture reactions during the so-called $r p$-process [6] while the heavier ones are created from $s$ and $r$-process seed nuclei by photodissociation processes like $(\gamma, n),(\gamma, p)$, and $(\gamma, \alpha)$ reactions in a process sometimes referred to as $\gamma$-process [5].

During the $\gamma$-process the $(\gamma, n)$ reactions compete with the $(\gamma, p)$ and $(\gamma, \alpha)$ processes if the $p$ and $\alpha$ separation energies of the produced proton-rich isotopes are low enough.

The special features corresponding to $p$-process nucleosynthesis in the $A \approx 100$ mass region are discussed in sect. 2. In sect. 3 the basics of Coulomb Dissociation experiments and the SIS/FRS/LAND setup at GSI Darmstadt are explained.

\footnotetext{
a e-mail: kerstin@ikp.tu-darmstadt.de
}

\section{The $A \approx 100$ mass region}

The three most abundant $p$ isotopes ( ${ }^{92} \mathrm{Mo}: 14.84 \%,{ }^{94} \mathrm{Mo}$ : $9.25 \%$, and ${ }^{96} \mathrm{Ru}: 5.52 \%$ natural elemental abundance) are found in the mass region $A \approx 100$. This region is the borderline between the $r p$ - and $\gamma$-process, i.e., it is not sure how the $p$ nuclei in this region are produced: in one of the two processes or in both simultaneously. Network calculations including only the $\gamma$-process fail to reproduce the observed abundances by a factor of 20 [5], thus, leading to the assumption that both processes are responsible for the production of the Mo and $\mathrm{Ru} p$ nuclei.

However, the difficulties in reproducing the observed abundances might also stem from the nuclear physics part: most of the reaction rates being involved in the network calculations (compare fig. 1) are calculated by HauserFeshbach statistical model calculations (e.g., [5]) due to the lack of experimental data in the astrophysically relevant energy region. The typical uncertainties of HauserFeshbach based calculations are about 20-30\% (e.g., [8]). However, if proton-rich nuclei in the vicinity of closed shells are concerned, it is not clear whether the involved level densities are high enough to legitimate this statistical approach. Thus, an experimental examination of the predicted reaction rates is highly desirable.

Different approaches are available and necessary to improve the experimental data base for the $\gamma$-process. While the $(\gamma, n)$ cross sections in the energy regime of the Giant Dipole Resonance around $15 \mathrm{MeV}$ have already been measured extensively several decades ago (see, e.g., [9]), the knowledge about the astrophysically relevant energy region close above the $n$ separation energy is rather scarce. 


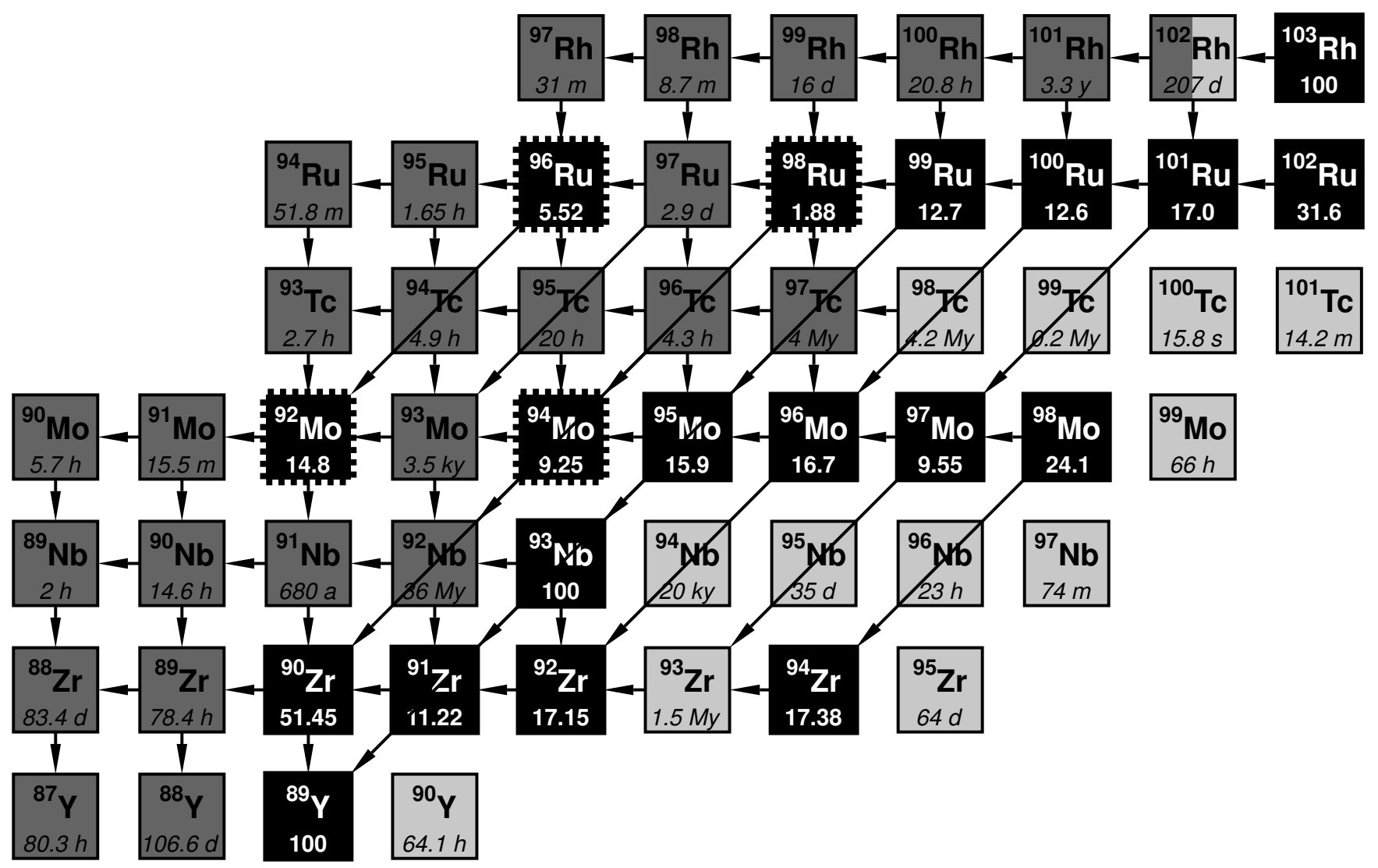

Fig. 1. Overview of the reactions involved in $p$-process nucleosynthesis in the mass region $A \approx 100$. The stable isotopes are printed in black boxes with their natural isotopic abundance. The $p$ nuclei ${ }^{92} \mathrm{Mo},{ }^{94} \mathrm{Mo},{ }^{96} \mathrm{Ru}$, and ${ }^{98} \mathrm{Ru}$ are marked with an indented margin. $\beta^{-}$-unstable isotopes are printed in light grey, the dark grey boxes stand for $\beta^{+}, \epsilon$-unstable isotopes both with their half-life under lab conditions. The relevance of the indicated $(\gamma, n),(\gamma, p)$, and $(\gamma, \alpha)$ reactions in a $p$-process network was calculated using ref. [7].

Some efforts using continuous bremsstrahlung spectra have been made at the S-DALINAC at Darmstadt $[10,11]$ and the ELBE setup at Rossendorf $[12,13]$ to determine the reaction rates without any assumptions on the shape of the cross section's energy dependence. A determination of the reaction rates by an absolute cross section measurement is possible using monoenergetic photon beams produced by Laser Compton Backscattering [14].

However, both methods are limited to stable target nuclei. Here, Coulomb dissociation (CD) of fast radioactive beams in the Coulomb field of a high- $Z$ target nucleus using virtual photons is a viable approach to measure the $(\gamma, n)$ cross sections indirectly. Such experiments can be performed with the SIS/FRS/LAND facility at GSI [15] as described in the following section.

The experimental knowledge about the $(\gamma, p)$ and $(\gamma, \alpha)$ reactions in the corresponding Gamow window is even worse. In fact, the experimental data is based on the observation of the time reversal $(p, \gamma)$ and $(\alpha, \gamma)$ cross sections, respectively $[16,17,18,19,20]$ for the proton-rich nuclei with mass numbers around 100. Due to the difficulties concerning the experimental accessibility of the $(\gamma, \alpha)$ reaction rates, a method using elastic $\alpha$ scattering has been established [21,22].
Likewise in the case of these charged particle reactions, CD provides an alternative possibility for experimental studies. The validity of the CD approach has been recently demonstrated for the case of the ${ }^{7} \operatorname{Be}(p, \gamma)$ reaction, where very good agreement has been found between direct and indirect methods [23,24].

\section{Coulomb dissociation experiments}

In Coulomb dissociation (CD) experiments the Coulomb field of a high- $Z$ nucleus is used to excite the nuclei one is interested in (see fig. 2). Thus, CD experiments are always done in inverse kinematics with the high- $Z$ nuclei as targets and the nuclei of interest as projectiles. This forced procedure yields the advantage that unstable isotopes can be observed if they are available as radioactive beams.

If, e.g., ${ }^{A} Z(\gamma, n)^{A-1} Z$ is the reaction of interest one has to study the reaction ${ }^{208} \mathrm{~Pb}\left({ }^{A} Z,{ }^{A-1} Z+n\right){ }^{208} \mathrm{~Pb}$ using $\mathrm{CD}$. The energy of the projectiles is choosen as high as possible due to several reasons. First of all, the higher the energy of the projectile the more the Coulomb field of the high- $Z$ nucleus is distorted, thus, leading to a higher fraction of $E 1$ excitations. Secondly, the used detection 


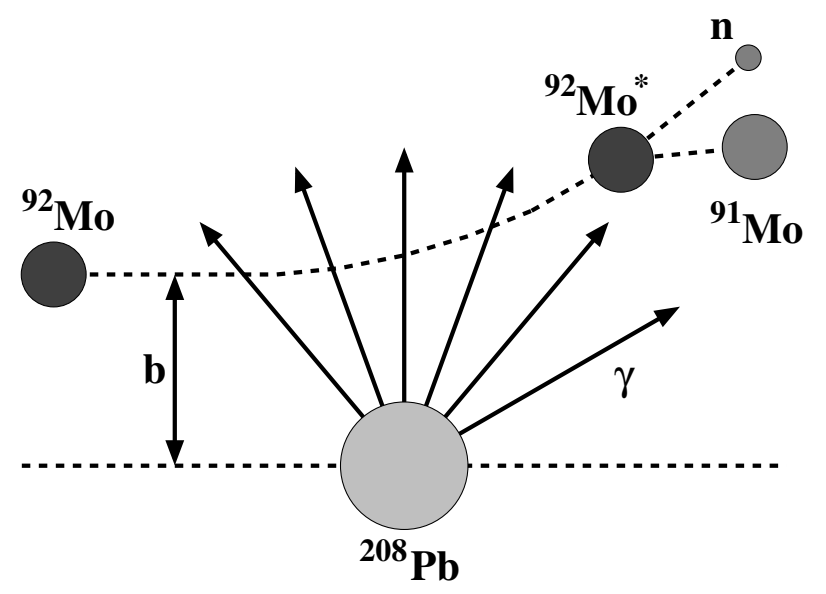

Fig. 2. Scheme of a Coulomb dissociation experiment in inverse kinematics observing the reaction ${ }^{92} \operatorname{Mo}(\gamma, n){ }^{91}$ Mo. The high- $Z$ target composed of ${ }^{208} \mathrm{~Pb}$ is shown with its surrounding field of virtual photons (marked with $\gamma$ ). The incoming ${ }^{92}$ Mo projectile has an impact parameter $b$. After passing the Coulomb field of ${ }^{208} \mathrm{~Pb}$ the now excited nucleus ${ }^{92} \mathrm{Mo}^{*}$ deexcites by emitting a neutron and remains as ${ }^{91} \mathrm{Mo}$.

systems cover $4 \pi$ solid angles due to the fact that all reaction products are predominantly focussed in forward directions. At last, CD dominates the nuclear background under small scattering angles, hence, yielding a possibility to distinguish between these two contributions to the cross section.

The experimental method used at GSI Darmstadt, is to produce a high-energy stable or radioactive beam and to measure the breakup products in secondary targets with full kinematics, thus, allowing the reconstruction of the excitation energy by utilizing the invariant-mass method. All projectile-like decay products are detected, i.e., all reaction products that have velocities close to the beam velocity. In this sense, the measurement is kinematically complete. Additionally, the $\gamma$-rays emitted by the excited projectile near the target position are measured.

The beam is delivered by the heavy-ion synchrotron SIS with energies up to $1 \mathrm{GeV} /$ nucleon and intensities of about $10^{10}$ ions/s depending on the accelerated nuclei. Radioactive beams are produced by the in-flight method using a Be production target with a thickness of $4 \mathrm{~g} / \mathrm{cm}^{2}$. The fragment separator FRS [25] is used to select the fragments of interest according to their magnetic rigidity. Furthermore, scintillation detectors are placed in the FRS beam-line to determine the masses of the fragments by time-of-flight measurements.

In front and behind the target the position of the beam is determined by Si pin-diodes. The dimension of the beam spot and its emittance is defined by a system of active slits called ROLU [26]. The $\mathrm{Pb}$ target is placed at the beginning of the CsI detector $[27,28]$ that is used to measure the $\gamma$-rays being emitted by the excited projectiles. Each of the single crystals covers a solid angle that is defined by the aim to realize the Doppler correction by suitable amplifications of the single signals. Thus, the energy of the emitted photon in the rest frame of the emitting source can be measured directly.

A large gap dipole magnet (ALADIN, see ref. [29]) separates the charged reaction products and the emitted neutrons. To determine the trajectories of the charged fragments, a position-sensitive Si pin-diode before the magnet and two large-area fiber detectors [30] behind the magnet are used. By defining the deflection angle in the magnetic dipole field, the magnetic rigidity of the particle is fixed. The velocity of the particles is measured with the timeof-flight (ToF) wall in combination with a thin organic plastic scintillator placed close to the target.

The LAND neutron detector [31] provides with its $2 \times 2 \mathrm{~m}^{2}$ active area a $100 \%$ acceptance for the emitted neutrons with kinetic energies up to $5.6 \mathrm{MeV}$. Using the two far-end sides of the one meter thick detector array timeof-flight and position information is available (description of the setup after [15]).

In the current experiment S295 the $(\gamma, n)$ cross sections of the isotopes ${ }^{92,93,94,100}$ Mo have been observed by CD. To study the stable isotopes ${ }^{94} \mathrm{Mo}$ and ${ }^{100} \mathrm{Mo}$ the corresponding beams were delivered by the synchrotron SIS. The beam of ${ }^{93} \mathrm{Mo}$ and ${ }^{92}$ Mo nuclei was produced by a primary ${ }^{94} \mathrm{Mo}$ beam via one and two neutron removal, respectively. However, the cross sections of these two isotopes have been measured one after the other due to an easier analysis.

Additionally, a measurement with a $\mathrm{C}$ and a Sn target instead of ${ }^{208} \mathrm{~Pb}$ was performed to subtract the nuclear contributions to the CD cross sections. To get full knowledge about the background conditions an emptytarget run was also carried out. The whole beam-time lasted for nine days and was realized by a collaboration of Forschungszentrum Rossendorf, Forschungszentrum Karlsruhe, GSI Darmstadt, and TU Darmstadt. The evaluation of the data has just started, hence, it is not yet possible to show preliminary results.

\section{Summary and outlook}

The $(\gamma, n)$ cross sections of the isotopes ${ }^{92,93,94,100}$ Mo have been measured using the Coulomb dissociation technique at the SIS/FRS/LAND setup at GSI Darmstadt. To establish the accuracy of this method, the cross sections of the stable isotopes ${ }^{92} \mathrm{Mo}$ and ${ }^{100} \mathrm{Mo}$ are also determined using real photons provided by the bremsstrahlung setups at ELBE and S-DALINAC, respectively.

The expected agreement of the results should establish the accuracy of the CD method. Once this goal is achieved, CD measurements on many critical but unstable nuclei for the $p$-process can be envisaged. A first example is the isotope ${ }^{93} \mathrm{Mo}$, that cannot be prepared as a target and was measured during the current experiment.

We thank the collaborators of experiment S295 from Forschungszentrum Rossendorf, Forschungszentrum Karlsruhe, and GSI Darmstadt for fruitful discussions and their support during beam-time. This work is supported by the DFG (contract SFB 634) and BMBF. 


\section{References}

1. F. Käppeler, Prog. Part. Nucl. Phys. 43, 419 (1999)

2. K.-L. Kratz, J.-P. Bitouzet, F.-K. Thielemann, P. Möller, B. Pfeiffer, Astrophys. J. 403, 216 (1993).

3. G. Wallerstein, I. Iben, P. Parker, A.M. Boesgaard, G.M. Hale, A.E. Champagne, C.A. Barnes, F. Käppeler, V.V. Smith, R.D. Hoffman, F.X. Timmes, C. Sneden, R.N. Boyd, B.S. Meyer, D.L. Lambert, Rev. Mod. Phys. 69, 995 (1997).

4. D.L. Lambert, V.V. Smith, M. Busso, R. Gallino, O. Straniero, Astrophys. J. 450, 302 (1995).

5. M. Arnould, S. Goriely, Phys. Rep. 384, 1 (2003).

6. H. Schatz, A. Aprahamian, J. Görres, M. Wiescher, T. Rauscher, J. Rembges, F.-K. Thielemann, B. Pfeiffer, P. Möller, K.-L. Kratz, H. Herndl, B. Brown, H. Rebel, Phys. Rev. 294, 167 (1998).

7. S. Goriely, Nuclear astrophysics data base (2003) http://www .astro.ulb.ac.be/Nucdata/.

8. T. Rauscher, F.-K. Thielemann, At. Data Nucl. Data Tables 75, 1 (2000).

9. B.L. Berman, S.C. Fultz, Rev. Mod. Phys. 47, 713 (1975).

10. K. Vogt, P. Mohr, M. Babilon, J. Enders, T. Hartmann, C. Hutter, T. Rauscher, S. Volz, A. Zilges, Phys. Rev. C 63, 055802 (2001).

11. K. Sonnabend, K. Vogt, D. Galaviz, S. Müller, A. Zilges, Phys. Rev. C 70, 035802 (2004).

12. M. Erhard et al., these proceedings.

13. G. Rusev et al., these proceedings.

14. H. Utsunomiya, H. Akimune, S. Goko, M. Ohta, H. Ueda, T. Yamagata, K. Yamasaki, H. Ohgaki, H. Toyokawa, Y.W. Lui, T. Hayakawa, T. Shizuma, S. Goriely, Phys. Rev. C 67, 015807 (2003).

15. R. Palit, P. Adrich, T. Aumann, K. Bortzky, B. Carlson, D. Cortina, U. Datta Pramanik, T. Elze, H. Emling, H. Geissel, M. Hellström, K. Jones, J. Kratz, R. Kulessa, Y. Leifels, A. Leistenschneider, G. Münzenberg, C. Nociforo, P. Reiter, H. Simon, K. Sümmerer, W. Walus, Phys. Rev. C 68, 034318 (2003).

16. T. Sauter, F. Käppeler, Phys. Rev. C 55, 3127 (1997).

17. J. Bork, H. Schatz, F. Käppeler, T. Rauscher, Phys. Rev. C 58, 524 (1998).

18. N. Özkan, A.S.J. Murphy, R.N. Boyd, A.L. Cole, M. Famiano, R.T. Güray, M. Howard, L. Şahin, J.J. Zach, R. deHaan, J. Görres, M.C. Wiescher, M.S. Islam, T. Rauscher, Nucl. Phys. A 710, 469 (2002).

19. W. Rapp, M. Heil, D. Hentschel, F. Käppeler, R. Reifarth, H.J. Brede, H. Klein, T. Rauscher, Phys. Rev. C 66, $015803(2002)$

20. G. Gyürky, Z. Fülöp, G. Kiss, Z. Máté, E. Somorjai, J. Görres, A. Palumbo, M. Wiescher, D. Galaviz, A. Kretschmer, K. Sonnabend, A. Zilges, T. Rauscher, Nucl. Phys. A 758, 517 (2005).
21. Z. Fülöp, G. Gyürky, Z. Máté, E. Somorjai, L. Zolnai, D. Galaviz, M. Babilon, P. Mohr, A. Zilges, T. Rauscher, H. Oberhummer, G. Staudt, Phys. Rev. C 64, 065805 (2001).

22. D. Galaviz, Z. Fülöp, G. Gyürky, Z. Máté, P. Mohr, T. Rauscher, E. Somorjai, A. Zilges, Phys. Rev. C 71, 065802 (2005).

23. F. Schümann, F. Hammache, S. Typel, F. Uhlig, K. Sümmerer, I. Böttcher, D. Cortina, A. Förster, M. Gai, H. Geissel, U. Greife, N. Iwasa, P. Koczoń, B. Kohlmeyer, R. Kulessa, H. Kumagai, N. Kurz, M. Menzel, T. Motobayashi, H. Oeschler, A. Ozawa, M. Ploskoń, W. Prokopowicz, W. Schwab, P. Senger, F. Strieder, C. Sturm, Z.-Y. Sun, G. Surówka, A. Wagner, W. Waluś, Phys. Rev. Lett. 90, 232501 (2003).

24. K. Sümmerer, these proceedings.

25. H. Geissel, P. Armbruster, K. Behr, A. Brünle, K. Burkard, M. Chen, H. Folger, B. Franczak, H. Keller, O. Klepper, B. Langenbeck, F. Nickel, E. Pfeng, M. Pfützner, E. Roeckl, K. Rykaczewski, I. Schall, D. Schardt, C. Scheidenberger, K.-H. Schmidt, A. Schröter, T. Schwab, K. Sümmerer, M. Weber, G. Münzenberg, T. Brohm, H.-G. Clerc, M. Fauerbach, J.-J. Gaimard, A. Grewe, E. Hanelt, B. Knödler, M. Steiner, B. Voss, J. Weckenmann, C. Ziegler, A. Magel, H. Wollnik, J. Dufour, Y. Fujita, D. Vieira, B. Sherrill, Nucl. Instrum. Methods B 70, 286 (1992).

26. G. Stengel, Entwicklung großflächiger Szintillatorfaserdetektoren und aktiver Blendensysteme, Diploma Thesis, Institut für Kernphysik, Universität Frankfurt, unpublished, 1996.

27. I. Kraus, Entwicklung eines CsI-Gammadetektors für Experimente mit radioaktiven Strahlen, Diploma Thesis, Institut für Kernphysik, Universität Frankfurt, unpublished, 1999.

28. T. Lange, Erprobung und Eichung eines CsI(Na)Gamma-Detektor-Systems für Experimente mit radioaktiven Strahlen, Diploma Thesis, Institut für Kernphysik, Universität Frankfurt, unpublished, 2001.

29. The ALADIN Collaboration, The forward spectrometer ALADIN at the $4 \pi$ detector, GSI Nachrichten 02-89, 1989.

30. J. Cub, G. Stengel, A. Grünschloß, K. Boretzky, T. Aumann, W. Dostal, B. Eberlein, T. Elze, H. Emling, J. Holeczek, R. Holzmann, G. Ickert, J. Kratz, R. Kulessa, Y. Leifels, H. Simon, K. Stelzer, J. Stroth, A. Surowiec, E. Wajda, Nucl. Instrum. Methods A 402, 67 (1998).

31. T. Blaich, T. Elze, H. Emling, H. Freiesleben, K. Grimm, W. Henning, R. Holzmann, G. Ickert, J. Keller, H. Klingler, W. Kneissl, R. Knig, R. Kulessa, J. Kratz, D. Lambrecht, J. Lange, Y. Leifels, E. Lubkiewicz, M. Proft, W. Prokopowicz, C. Schütter, R. Schmidt, H. Spies, K. Stelzer, J. Stroth, W. Walús, E. Wajda, H. Wollersheim, M. Zinser, E. Zude, Nucl. Instrum. Methods A 314, 136 (1992). 